\title{
SOME REMARKS ON THE STABILITY OF THE CRITERIA FOR OCCURRENCE OF MAXIMUM AVAILABILITY IN THE OPERATION SYSTEM
}

\author{
M. Szubartowski ${ }^{*}$, L. Knopik ${ }^{* *}$, K. Migawa ${ }^{* * *}$, A. Soltysiak ${ }^{\dagger}$
}

\begin{abstract}
The paper examines a 3-state system of preventive replacements. It was shown that the system availability for which failure time has distribution only slightly different from the exponential distribution does not reach maximum value.
\end{abstract}

Keywords: reliability function, failure rate function, availability, age replacement

\section{Introduction}

Industrial system management requires implementation of various operational activities. One of the important tasks in which the role of optimization will grow is the maintenance of operation system including replacement of technical objects. However, maintenance and replacement are not a merely technical question as they are an economical one. The strategy of maintaining the focus on preventive maintenance is implemented mainly in the area of operational research and management studies. The replacement strategy according to the age of technical objects relies on the object's preventive replacement upon reaching age $T$, or corrective one (failure) before reaching age $T$. Preventive replacements are less expansive and cheaper than corrective replacements. The time of preventive replacement is usually known to be shorter than corrective replacement time. Fundamental facts for age replacement are included in papers (Barlow et al., 1965 and Barlow et al., 1960). Preventive replacements are introduced in order to increase the availability of machine operation systems as well as profit per unit of time of the operation system. The paper analyzes the model of technical object age replacement for a 3-state system. The criteria function used in the paper expresses the availability coefficient. The probability distribution for technical object failure time is assumed to be known, while the preventive exchange strategy will be implanted in the second time interval. The problem of profit maximization per time unit as well as availability for growing failure rate functions or a wider intensity function class are examined.

The paper examines 3-state system with states $S_{1}, S_{2}$ i $S_{3}$, where $S_{1}$ is the state of correct operation (utilization), $S_{2}$ is the state of repair (renewal), and $S_{3}$ is the state of preventive servicing. The state changes are assumed to be defined by stochastic process $X(t)$. If $X(t)=i$, where $i=1,2,3$, then the technical object at moment $t$ is at the state $i$. The profit per time unit resulting from the technical object remaining at state $i$ is marked by $z_{i}, i=1,2,3$. The matrix of transfer probabilities embedded in $X(t)$

RNDr. Mirosław Szubartowski, DSc.: Management Business and Service, Fordońska Street 40, 85-719 Bydgoszcz, Poland, analityk@karor.com.pl

** Assoc. Prof. Leszek Knopik, PhD.: Faculty of Management, UTP University of Science and Technology, Fordońska Street 430, 85-790 Bydgoszcz, Poland, knopikl@utp.edu.pl

*** Assoc. Prof. Klaudiusz Migawa, PhD.: Faculty of Mechanical Engineering, UTP University of Science and Technology, Al. prof. S. Kaliskiego 7, 85-789 Bydgoszcz, Poland, klaudiusz.migawa@utp.edu.pl

† Agnieszka Sołtysiak, MSc.: Faculty of Mechanical Engineering, UTP University of Science and Technology, Al. prof. S. Kaliskiego 7, 85-789 Bydgoszcz, Poland, agnieszka.soltysiak@utp.edu.pl 
process of Markov chain is marked by $P=\left[p_{i j}\right] . T_{i}$ stands for the time of the object remaining in the state $S_{i}, F_{i}(t)$ stands for the distribution function, $R_{i}(t)$ stands for the reliability function, $f_{i}(x)$ stands for probability density, $\lambda_{i}(t)$ stands for function of failure intensity against failure $T_{i}$. In the paper (Knopik, 2010), taking into consideration certain general assumptions, it was demonstrated that the criteria function $g(x)$ expressing profit per time unit, is shown through the formula:

$$
g(x)=\frac{A z_{1} E T_{1}(x)+B_{1} F_{1}(x)+C_{1}}{A E T_{1}(x)+B F_{1}(x)+C}
$$

where $x$ is the moment of the exchange of an element (technical object).

From the paper (Knopik, 2010), the coefficients $A, B, B_{1}, C, C_{1}$ of the formula (1) are known to be written in the following form:

$$
\begin{gathered}
A=p_{12} p_{32}+p_{31} \\
B=p_{12}\left(z_{2} E T_{2} p_{31}-z_{3} E T_{3} p_{21}\right) \\
B_{1}=p_{12}\left(E T_{2} p_{31}-E T_{3} p_{21}\right) \\
C=z_{2} E T_{2} p_{12}+z_{3} E T_{3} \\
C_{1}=E T_{2} p_{12}+E T_{3}
\end{gathered}
$$

The symbol $E T_{1}(x)$ stands for the integral in the following form:

$$
E T_{1}(x)=\int_{0}^{x} R_{1}(t) d t
$$

Derivative of criteria function $g(x)$ is expressed by the formula:

$$
g^{\prime}(x)=\frac{1}{M^{2}(x)}\left(A \alpha r_{1}(x)+\beta R_{1}(x)+f_{1}(x) \gamma\right)
$$

where $M(x)$ is the denominator of the formula (1).

Coefficients $\alpha, \beta, \gamma$ are thus expressed in the relation to the parameters of the system:

$$
\begin{gathered}
\alpha=A p_{12}\left[E T_{2} p_{31}\left(z_{2}-z_{1}\right)+E T_{3} p_{21}\left(z_{1}-z_{3}\right)\right] \\
\beta=A\left[E T_{2} p_{32}\left(z_{1}-z_{2}\right)+E T_{3}\left(z_{1}-z_{3}\right)\right] \\
\gamma=A E T_{2} E T_{3}\left(z_{2}-z_{3}\right)
\end{gathered}
$$

The paper assumes the following:

Z1. $z_{1}>0, z_{2}<0, z_{3}<0$,

Z2. $z_{2}<z_{3}$,

Z3. $E T_{2} p_{31} \geq E T_{3} p_{32}$.

The above assumptions allow one to formulate the inequality: $\alpha<0, \beta>0, \gamma \leq 0$. These inequalities play a crucial role in formulating the sufficient condition for the occurrence of maximum criteria function $g(x)$.

The sing of the derivative $g^{\prime}(x)$ is the same as the sign of the function:

$$
h_{2}(x)=\alpha H(x)+\beta R_{1}(x)+\gamma f_{1}(x)
$$

where: $H(x)=f_{1}(x) E T_{1}(x)-F_{1}(x) R_{1}(x)$.

In order to simplify the markings in the following part of our paper, it is assumed that $f_{1}(x)=f(x)$, $F_{1}(x)=F(x), R_{1}(x)=R(x), \lambda_{1}(x)=\lambda(x)$. By substituting $z_{1}=1, z_{2}=0, z_{3}=0$ in the formula (1), availability is obtained. In this paper the availability is analyzed as a criteria function. The considerations for the criteria function determining profit per unit are similar.

The basic property of the function $H(x)=f(x) E T(x)-F(x) R(x)$ are included in the conclusions:

Conclusion 1. $H(0)=0, H(\infty)=0$.

Conclusion 2. $H(x)=0$ for $x \geq 0$ if, and only if the random variable $T$ has exponential distribution.

The derivative of availability has the same sign as the function symbol:

$$
h(x)=\alpha[\lambda(x) E T(x)-F(x)]+\beta
$$

where: $\lambda(x)=\frac{f(x)}{R(x)}$.

Let $h_{1}(x)=\lambda(x) E T(x)-F(x)$. The basis for the property of function $h_{1}(x)$ is formed below.

Conclusion 3. $h_{1}(0)=0, h_{1}(\infty)=\lambda(\infty) E T-1$. 
Conclusion 4. $h_{1}(x)=0$ for $x \geq 0$ if, and only if time before failure $T$ has exponential distribution.

Conclusion 5. If the derivative $\lambda^{\prime}(x)$ of failure rate function exists, the equality $h_{1}^{\prime}(x)=\lambda^{\prime}(x) E T(x)$ is valid.

In the assumption for the final conclusion, a differentiable function of probability density $f(x)$ is sufficient. Conclusion 5 results in function $h_{1}(x)$ reaching maximum and minimum values in the same points as function $\lambda(x)$.

\section{Sufficient conditions for occurrence of maximum availability}

A number of sufficient conditions for the occurrence of maximum availability $g(x)$ are formed below.

Conclusion 6. If $T \in I F R$ (Increasing Failure Rate), $h(\infty)<0$, then availability $g(x)$ reaches maximum at a certain point.

Proof. The growing function $h(x), h(0)=\beta>0$ and $h(\infty)<0$ results in the derivative $g^{\prime}(x)$ growing and changing its sign + to - . Hence $g(x)$ takes the maximum value. Some time distributions have the characteristic $\lambda(\infty)=\infty$. Such distributions include Weibull distribution.

Conclusion 7. If $T \in I F R, \lambda(\infty)=\infty$, then $g(x)$ reaches an exactly one maximum.

However, for certain distributions $\lambda(\infty)<\infty$ occurs. Such distributions include the gamma distribution. The random variable $T$ has a gamma distribution if its density is expressed through the formula:

$$
f(x)=\frac{b^{p}}{\Gamma(p)} x^{p-1} e^{-b x}
$$

for $x>0, p>0, b>0 . \Gamma(p)$ stands for a gamma function defined by the formula:

$$
\Gamma(p)=\int_{0}^{\infty} x^{p-1} e^{-x} d x
$$

where $p$ as well as $b$ are distribution parameters.

Symbolically, it is written: $T \sim \Gamma(p, b)$. It is known that if $p>1$, then $T \in \operatorname{IFR}$ and $\lambda(\infty)=b$.

Conclusion 8. If time before failure $T$ has distribution $\Gamma(p, b)$, so that $p>1-\frac{\beta}{\alpha}$, then availability $g(x)$ reaches an exactly one maximum. In the papers (Knopik, 2005 and Knopik, 2006) a wider lifetime class than IFR is introduced, called MTFR.

Definition. $T \in M T F R$, if $H(x) \geq 0$.

The MTFR class includes some distributions with unimodal failure rate function.

Conclusion 9. If $h_{1}(x) \geq 0$ for $x \geq 0, \lambda(x)$ is a unimodal function and $h_{1}(\infty)=\lambda(\infty) E T-1 \geq 0$, then $T \in M T F R$.

The sufficient condition for occurrence of exactly one maximum of availability form a unimodal failure intensity function is formulated in conclusion 10.

Conclusion 10. If $\lambda(x)$ is unimodal, $T \in M T F R, h(\infty)<0$, the availability reaches an exactly one maximum.

\section{Stability of sufficient conditions for occurrence of maximum}

Conclusion 2 leads to random variable $T$ having exponential distribution, $h(x)=\beta>0$. As a result, the criteria function $g(x)$ grows. It should be assumed that, for distributions "close" to exponential distribution, function $g(x)$ also does not reach maximum value. In research connected with the impact of disturbances in exponential distribution on the criteria of the occurrence of maximum availability, the results of research from the area of stability of the characteristics of exponential distribution may be helpful. For example, paper (Azlarov et al., 1986) shows that the following implication is valid:

$$
\text { if }|\lambda(x)-\lambda|<\varepsilon \text {, then }|R(x)-\exp (-\lambda x)|<\frac{\varepsilon \lambda}{1-\varepsilon}
$$

where $0<\varepsilon<1, E T=\frac{1}{\lambda}$.

The previous implication shows that small disturbances of failure rate function $\lambda(x)$ of random variable $T$ lead to minor disturbances of reliability function $R(x)$. The current state of research on the 
characterization of stability of exponential distribution were presented in the paper (Roy et al., 2013). In research of stability of the criteria of occurrence of maximum availability the following form of implication is helpful:

$$
\text { if }|\lambda(x)-\lambda|<\varepsilon \text {, then }|H(x)|<\phi(\varepsilon)
$$

where $\phi(\varepsilon)$ is a certain function, $E T=\frac{1}{\lambda}$. However, the authors of this paper are not familiar with the form of the function $\phi(\varepsilon)$. On the basis of the given disturbance of function $\lambda(x)$ a certain realization of function $\phi(\varepsilon)$ is shown below. Let $\lambda(x)=\lambda-\frac{\varepsilon}{x+1}$, then the accumulated function of failure rate has the form:

$$
\Lambda(x)=\lambda x-\varepsilon \ln (x+1)
$$

The reliability function:

$$
R(x)=\exp (-\lambda x)(x+1)^{\varepsilon}
$$

In order for the function $R(x)$ to become a reliability function, $0<\varepsilon<\lambda$ is required. For the function $E T(x)$ described with formula (2) we implement integration by parts:

$$
E T(x)=\frac{1}{\lambda}\left(1-e^{-\lambda x}(x+1)^{\varepsilon}\right)+\frac{\varepsilon}{\lambda} \int_{0}^{x} e^{-\lambda t}(t+1)^{\varepsilon-1} d t
$$

Below, an evaluation of the function $h_{1}(x)=\lambda(x) E T(x)-F(x)$, from up and down is constructed:

$$
h_{1}(x)=\varepsilon \int_{0}^{x} e^{-\lambda t}(t+1)^{\varepsilon-1} d t-\frac{\varepsilon}{x+1}\left[\frac{1}{\lambda}\left(1-e^{-\lambda x}(x+1)^{\varepsilon}\right)+\frac{\varepsilon}{\lambda} \int_{0}^{x} e^{-\lambda t}(t+1)^{\varepsilon-1} d t\right](12)
$$

The inequality $h_{1}(x) \geq 0$ is equivalent to the inequality:

$$
g(x)=(\lambda(x+1)-\varepsilon) \int_{0}^{x} e^{-\lambda t}(t+1)^{\varepsilon-1} d t-1+e^{-\lambda x}(x+1)^{\varepsilon}
$$

Derivative $g^{\prime}(x)$ of the function $g(x)$ may be written as:

$$
g^{\prime}(x)=\lambda \int_{0}^{x} e^{-\lambda t}(t+1)^{\varepsilon-1} d t
$$

Since $g(0)=0$ and $g^{\prime}(x) \geq 0$ for $x \geq 0, h_{1}(x) \geq 0$ for $x \geq 0$. This means that random variable $T$ with reliability function $R(x)$ belongs to MTFR class. The following inequality results from formula (12) for $0<\varepsilon<1$ :

$$
h_{1}(x) \leq \varepsilon \int_{0}^{x} e^{-\lambda t}(t+1)^{\varepsilon-1} d t
$$

The previous inequality and the fact that $0<\varepsilon<1$ result in $h_{1}(x)<\frac{\varepsilon}{\lambda}$. For sufficiently small $\varepsilon$ and for $x \geq 0$ the following inequality is valid:

$$
\alpha h_{1}(x)+\beta=\alpha \frac{\varepsilon}{\lambda}+\beta>0
$$

Conclusion 11 . For sufficiently small $\varepsilon$ the criteria function $g(x)$ grows.

\section{Conclusions}

The previous conclusion allows us to assume that in order for the criteria function $g(x)$ to reach the maximum value, it is necessary for the time distribution before failure $T$ to be "decisively" different from the exponential distribution.

\section{References}

Azlarov, T. A. and Volodin, N. A. (1986) Characterization Problems Associated with the exponential distribution. Springer Verlag.

Barlow, R. E. and Prochan, F. (1965) Mathematical Theory of Reliability. John Wiley \& Sons.

Barlow, R. E. and Hunter, L. (1960) Optimum preventive maintenance polices. Operation Research, vol. 8, pp. 90100.

Knopik, L. (2005) Some results of a class of lifetime distributions. Control and Cybernetics, 35(2), pp. 1-8.

Knopik, L. (2006) Characterization of a class of lifetime distribution. Some results of a class of lifetime distributions. Control and Cybernetics, 34(1), pp. 407-414.

Knopik, L. (2010) Method of choice efficiency strategy of the maintenance of technical object. University of Science and Technology in Bydgoszcz, Publishing Department, Dissertations no. 145.

Roy, D. and Roy, R. (2013) Stability of the characterization results in terms of hazard rate and mean residual life for the univariate and bivariate setups. Communications in Statistics - Theory and Methods, vol. 42, pp. 1583-1598. 